\title{
AN EXAMPLE OF NORMAL LOCAL RING WHICH IS ANALYTICALLY RAMIFIED
}

\author{
MASAYOSHI NAGATA
}

Previously the following question was offered by Zariski [6]:

Is any normal Noetherian local ring analytically irreducible $?^{1}$

In the present note, we will construct a counter-example against the question.

Terminology. A ring (integrity domain) means always a commutative ring (integrity domain) with identity. A normal ring is an integrity domain which is integrally closed in its field of quotients. When $\mathfrak{D}$ is an integrity domain, the integral closure of $\mathfrak{D}$ in its field of quotients is called the derived normal ring of 0 .

In our treatment, some basic notions and results on general commutative rings and Noetherian local rings are assumed to be well known (see, for example, [5] and one of [1] or [2]). In particular, some results on regular local rings and completions of local rings are used freely (without references). On the other hand, we will make use of an example constructed in $[3, \S 1]$ without proof.

\section{$\S 1$. The construction of an example}

Let $\mathbf{k}_{0}$ be a perfect field of characteristic 2 and let $u_{0}, v_{0}, \ldots, u_{n}, v_{n}, \ldots$ (infinitely many) be algebraically independent elements over $\mathbf{k}_{0}$. Set $\mathbf{k}=\mathbf{k}_{0}\left(\boldsymbol{u}_{0}\right.$, $\left.v_{0}, \ldots, u_{n}, v_{n}, \ldots\right)$. Further let $x$ and $y$ be indeterminates and set $\mathfrak{r}=\mathbf{k}\{x, y\}$ (formal power series ring), $\mathfrak{o}=\mathbf{k}^{2}\{x, y\}[\mathbf{k}]$ and $c=\sum_{i=0}^{\infty}\left(u_{i} x^{i}+v_{i} y^{i}\right)$. Then we set $2=0[c]$.

Proposition. $\mathfrak{i}$ is a normal Noetherian local ring and the completion of $\mathcal{z}$ contains non-zero nilpotent elements (that is, $\$$ is analytically ramified).

Received January 10, 1955.

1) It was conjectured that the answer is negative by [4] and the present paper answers the conjecture affirmatively. 


\section{$\S 2$. Some preliminary results}

Lемма 1. The ring $\mathfrak{D}$ is a regular local ring with a regular system of parameters $x, y, \mathfrak{r}$ is the completion of $\mathrm{D}$.

For the proof, see $[3, \S 1]$.

LEMma 2. An element $\sum a_{i j} x^{i} y^{j}\left(a_{i j} \in \mathbf{k}\right)$ is in $\mathfrak{o}$ if and only if $\left[\mathbf{k}^{2}\left(a_{00}, a_{01}\right.\right.$, $\left.\left.a_{10}, \ldots\right): \mathbf{k}^{2}\right]$ is finite.

Proof. $b=\sum a_{i j} x^{i} y^{j}$ is in $\mathfrak{D}$ if and only if $b$ is in $\mathbf{k}^{2}\{x, y\}\left[u_{0}, v_{0}, \ldots\right.$, $\left.u_{n}, v_{n}\right]$ for some $n$. Therefore we see our assertion easily.

Lemma 3. Set $d_{n}=\sum_{i=0}^{\infty} u_{n+i} x^{i}, e_{n}=\sum_{i=0}^{\infty} v_{n+i} y^{i}(n=0,1, \ldots) . \quad$ Then $\mathrm{t}=\mathrm{D}\left[d_{0}\right.$, $\left.e_{0}, \ldots, d_{n}, e_{n}, \ldots\right]$ is a normal ring.

Proof. Let $f$ be any element of the derived normal ring of t. Since $f$ is in the field of quotients of $t, f$ is expressed in the form $\left(p+q d_{n}+r e_{n}+s e_{n} d_{n}\right) / t$ $\langle p, q, r, s, t \in \mathfrak{D}, t \neq 0)$ (because $\mathfrak{D}\left[d_{0}, e_{0}, \ldots, d_{n}, e_{n}\right]=\mathfrak{D}\left[d_{n}, e_{n}\right]$ by the construction). Since $p, q, r, s$ and $t$ are in $n$, there exists an integer $N$ which is not less than $n$ such that the coefficients of them (as the power series in $x$ and $y)$ are in $\mathbf{k}^{2}\left(u_{0}, v_{0}, \ldots, u_{N-1}, v_{N-1}\right)$. Then since $d_{n}=u_{n}+u_{n+1} x+\ldots$ $+u_{N-1} x^{N-n-1}+x^{v-n} d_{N}$ and $e_{n}=v_{n}+\ldots+v_{N-1} y^{N-n-1}+y^{N-n} e_{N}, f$ is in the derived normal ring of $\mathfrak{D}^{*}\left[d_{N}, e_{N}\right]$, where $\mathfrak{D}^{*}=\mathbf{k}^{2}\{x, y\}\left[u_{0}, v_{0}, \ldots, u_{N-1}, v_{N-1}\right]$ (because $p, q, r, s$ and $t$ are in $D^{*}$ by our assumption and because the square of $f$ is in $\left.\mathbf{k}^{2}\{x, y\} \subseteq \mathfrak{o}^{*}\right)$. Since the maximal ideal of $\mathrm{D}^{*}$ is generated by $x$ and $y$, as is easily seen, $\mathfrak{D}^{*}$ is a (complete) regular local ring. Since the residue class field of $\mathrm{D}^{*}$ is represented by $\mathbf{k}^{2}\left(u_{0}, v_{0}, \ldots, u_{N-1}, v_{N-1}\right)$ and since the leading forms of $d_{N}$ and $e_{N}$ are $u_{N}$ and $v_{N}$ (respectively), the maximal ideal of $0^{*}\left[\mathrm{~d}_{N}, e_{N}\right]$ is generated by $x$ and $y$. Therefore $\mathfrak{D}^{*}\left[d_{v}, e_{x}\right]$ is a regular local ring and is a normal ring. Therefore $f$ is in $D^{*}\left[d_{v}, e_{x}\right]$ and therefore $f$ is in $t$. Thus we see that $t$ is normal.

LEMMA 4. $x$ ? and $y$ ? are prime ideals.

Proof. $\sum_{2} / x_{2}$ is isomorphic to $\mathbf{k}^{2}\{y\}[\mathbf{k}]\left[e_{0}\right]$, which is an integrity domain. Therefore $x$ is a prime ideal. That $y$ is prime follows similarly.

Leмma 5. Let $\mathfrak{g}^{\prime}$ be the derived normal ring of $z$ and let $f$ be an element

2) By virtue of this result, we see easily that $t$ is a regular local ring. 


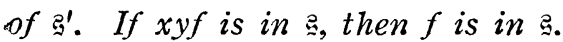

Proof. Since $\mathfrak{D}$ is Noetherian and since $z=\mathfrak{D}[c], ?$ is Noetherian. Therefore if $f$ is not in $\xi$, then one of the following must hold (see $[5, \S 8]$ ): 1) $x y$ g has an imbedded prime divisor; 2) there exists at least one minimal prime divisor $p$ of $x y$ such that $\mathfrak{y}_{p}$ is not normal. Both are impossible because $x \mathfrak{y}^{2}$ and $y \mathfrak{z}$ are prime ideals by Lemma 4. Thus we see that $f$ is in $\stackrel{3}{\text {. }}$

\section{$\S 3$. Proof of the proposition}

As was noted above, $\mathfrak{Z}$ is Noetherian. Since $\mathcal{g}$ is isomorphic to $\triangleright[X] / g(X) \vee[X]$, where $g(X)=X^{2}-c^{2}$, the completion of $g$ is isomorphic to $\mathfrak{r}[X] / g(X) \mathfrak{r}[X]$ (because $\mathfrak{r}$ is the completion of $\mathfrak{o}$ ). The residue class of $X-c$ is not zero and is nilpotent. Therefore the completion of $g$ contains non-zero nilpotent elements. Now we will show that $\mathfrak{g}$ is normal. Let $f$ be any element of $\mathfrak{g}^{\prime}$. Since $\mathfrak{z}$ is contained in $\mathrm{t}$ (because $c=d_{0}+e_{0}$ ) and since $t$ is normal by Lemma $3, f$ is in $t$. Therefore $f$ is of the form $p+q d_{n}+r e_{n}+s d_{n} e_{n}(p, q, r, s \in \mathfrak{0})$. Then $x^{n} y^{n} f$ is in $\mathfrak{D}\left[d_{0}, e_{0}\right]$. In order to show that $f$ is in $\mathfrak{g}$, we have only to show that $x^{n} y^{n} f$ is in $g$ by Lemma 5 . Therefore we may assume that $n=0$. Since $f$ is in the field of quotients of $z, f$ is of the form $(t+u c) / v$ $(t, u, v \in \mathfrak{0})$. Since $c=d_{0}+e_{0}$, we see that $(t / v)+(u / v) d_{0}+(u / v) e_{0}=p+q d_{0}$ $+r e_{0}+s d_{0} e_{0}$. Since $1, d_{0}, e_{0}, d_{0} e_{0}$ are linearly independent over $\mathfrak{v}$, we have $t^{\prime} v$ $=p, u / v=q(=r, s=0)$. Therefore $f=p+q c$, which is in $\mathfrak{g}_{\text {. Therefore } z \text { is a }}$ normal ring.

\section{BIBLIOGRAPHY}

[1] C. Chevalley, On the theory of local rings, Ann. of Math., vol. 44 (1943), pp. 690-708.

[2] I. S. Cohen, On the structure and ideal theory of complete local rings, Trans. Amer. Math. Soc., vol. 59 (1946), pp. 54-106.

[3] M. Nagata, Note on integral closures of Noetherian domains, Memoirs Kyoto, Ser. A., vol. 28 (1953), pp. 121-124.

[4] M. Nagata, On the theory of Henselian rings, II, Nagoya Math. Journ., vol. 7 (1954), pp. 1-19.

[5] M. Nagata, Basic theorems on general commutative rings, to appear in Memoirs Kyoto, Ser. A.

[6] O. Zariski, Analytical irreducibility of normal varieties, Ann. of Math., vol. 49 (1948), pp. 352-361.

Mathematical Institute, Kyoto University 